\section{OPEN ACCESS}

Edited by: Tomoyuki Kuwaki, Kagoshima University, Japan

Reviewed by: Wladyslaw-Lason, Institute of Pharmacology (PAS), Poland

Yukihiro Ohno, Osaka University of Pharmaceutical Sciences, Japan

*Correspondence: Yoshitoshi Kasuya kasuya@faculty.chiba-u.jp

Specialty section: This article was submitted to

Neuropharmacology,

a section of the journal

Frontiers in Pharmacology

Received: 13 December 2016 Accepted: 06 February 2017 Published: 17 February 2017

Citation:

Umezawa H, Naito Y, Tanaka K, Yoshioka $K$, Suzuki $K$, Sudo T, Hagihara M, Hatano M, Tatsumi K and Kasuya Y (2017) Genetic and Pharmacological Inhibition of p38a Improves Locomotor Recovery after Spinal Cord Injury. Front. Pharmacol. 8:72. doi: 10.3389/fphar.2017.00072

\title{
Genetic and Pharmacological Inhibition of p38 $\alpha$ Improves Locomotor Recovery after Spinal Cord Injury
}

\begin{abstract}
Hiroki Umezawa 1,2,3, Yusuke Naito ${ }^{1,2,3}$, Kensuke Tanaka ${ }^{1,2}$, Kento Yoshioka ${ }^{2,3}$, Kenichi Suzuki 1,3, Tatsuhiko Sudo ${ }^{4}$, Masahiko Hagihara ${ }^{5}$, Masahiko Hatano ${ }^{3}$, Koichiro Tatsumi ${ }^{1}$ and Yoshitoshi Kasuya ${ }^{2,3 *}$
\end{abstract}

\begin{abstract}
'Department of Respirology, Graduate School of Medicine, Chiba University, Chiba, Japan, ${ }^{2}$ Department of Biochemistry and Molecular Pharmacology, Graduate School of Medicine, Chiba University, Chiba, Japan, ${ }^{3}$ Department of Biomedical Science, Graduate School of Medicine, Chiba University, Chiba, Japan, ${ }^{4}$ Chemical Biology Core Facility and Antibiotics Laboratory, RIKEN Advanced Science Institute, Saitama, Japan, ${ }^{5}$ Corporate Research \& Development, Ube Industries, Ltd, Ube, Japan
\end{abstract}

One of the mitogen-activated protein kinases, p38 $\alpha$ plays a crucial role in various inflammatory diseases and apoptosis of various types of cells. In this study, we investigated the pathophysiological roles of p38 $\alpha$ in spinal cord injury (SCl), using a mouse model. Lateral hemisection at T9 of the SC was performed in wild type (WT) and p38 $\alpha^{+/-}$mice (p38 $\alpha^{-1-}$ showed embryonic lethality). p38 $\alpha^{+/-}$mice showed a better functional recovery from SCl-associated paralyzed hindlimbs compared to WT mice at 7 days post-injury (dpi), which remained until 28 dpi (an end time point of monitoring the behavior). In histopathological analysis at $28 \mathrm{dpi}$, there was more axonal regeneration with remyelination on the caudal side of the lesion epicenter in $\mathrm{p} 38 \alpha^{+/-}$mice than in WT mice. At $7 \mathrm{dpi}$, infiltration of inflammatory cells into the lesion and expression of cytokines in the lesion were reduced in p38 $\alpha^{+/-}$mice compared with WT mice. At the same time point, the number of apoptotic oligodendrocytes in the white matter at the caudal boarder of the lesion of p38 $\alpha^{+/-}$mice was lower than that of WT mice. At $14 \mathrm{dpi}$, more neural and oligodendrocyte precursor cells in the gray matter and white matter, respectively, were observed around the lesion epicenter of $\mathrm{p} 38 \alpha^{+/-}$mice compared with the case of WT mice. At the same time point, astrocytic scar formation was less apparent in $\mathrm{p} 38 \alpha^{+/-}$than in WT mice, while compaction of inflammatory immune cells associated with the wound contraction was more apparent in p38 $\alpha^{+/-}$than in WT mice. Furthermore, we verified the effectiveness of oral administration of SB239063, a p38a inhibitor on the hindlimb locomotor recovery after SCl. These results suggest that $\mathrm{p} 38 \alpha$ deeply contributes to the pathogenesis of $\mathrm{SCl}$ and that inhibition of p38 $\alpha$ is a beneficial strategy to recovery from $\mathrm{SCl}$.

Keywords: p38 mitogen-activated protein kinases, spinal cord injury (SCI), recovery of locomotor activity, tissue degeneration, tissue regeneration 


\section{INTRODUCTION}

Spinal cord injury (SCI) results in limited motor function recovery under the chronic phase, mainly because of the poor regenerative capability of adult mammalian central nervous system (CNS) (Horner and Gage, 2000). SCI is composed of three phases, acute, secondary and chronic, and its outcomes are influenced by the secondary phase (Oyinbo, 2011). The secondary phase is characterized by inflammation-triggered events as follows: edema, apoptosis of cells including neurons and oligodendrocytes, demyelination, astrocytic scar formation and so on (Zhou et al., 2014). Under the secondary phase of SCI, gradual functional recovery is observed in several animals including humans, the extent of which is inversely related to the intensity of primary damage (Becker et al., 2003). It is thus logical to postulate that reduction of secondary damage waves by controlling inflammation-triggered events may improve the functional recovery after SCI.

p38 is one of mitogen-activated protein kinases (MAPKs) which transduces a variety of extracellular signals to the transcriptional machinery. By using genetically engineered mice, it has been demonstrated that p38 participates at least in inflammatory responses and cell fate decision including apoptosis (O'Keefe et al., 2007; Ventura et al., 2007; Kang et al., 2008; Risco et al., 2012). Among four mammalian isoforms of p38 $(\alpha, \beta, \gamma$, and $\delta), \mathrm{p} 38 \alpha$ is expressed ubiquitously in adult tissues and functions as a central player of p38 isoforms (Kumar et al., 2003). Although $p 38 \alpha$ gene (MAPK14) deficiency results in lethality in homozygous embryonic mice, the p38 $\alpha^{+/-}$ mouse is a useful tool for studying the in vivo role of $\mathrm{p} 38 \alpha$ in certain disease models (Tamura et al., 2000; TakanamiOhnishi et al., 2002; Matsuo et al., 2006). In particular, p38 $\alpha^{+/-}$ mice show an outstanding resistance to neurodegenerative diseases such as epileptic seizure and experimental autoimmune encephalomyelitis (EAE, an animal model of multiple sclerosis associated with demyelination in the SC) (Namiki et al., 2007, 2012). In contrast, the deterioration of EAE was observed in transgenic mice expressing a constitutive active form of MKK6, a p38-specific activator (Noubade et al., 2011). Furthermore, self-renewal activity and neural differentiation capacity of neural stem cells (NSCs) in the hippocampus of $\mathrm{p} 38 \alpha^{+/-}$mice are higher than those of WT mice (Yoshioka et al., 2015). These previous findings tempt us to think that inhibition of p38 $\alpha$ may be beneficial to the functional recovery after SCI. In fact, it has been demonstrated that a p38 $\alpha$ inhibitor, SB203580 could reduce the damage of hindlimb function after SCI (Horiuchi et al., 2003). In contrast, another group showed that SB203580 failed to improve functional outcome after SCI (Stirling et al., 2008). Those reports are fully controversial though employing a similar SCI protocol and a same administration procedure. Therefore, whether $\mathrm{p} 38 \alpha$ is recognized as a potential therapeutic target in $\mathrm{SCI}$ is still under debate.

Here, we first showed that the hindlimb locomotor behavior was improved in $\mathrm{p} 38 \alpha^{+/-}$mice compared to WT mice. As the mechanisms underlying the improved signs of SCI in $\mathrm{p} 38 \alpha^{+/-}$ mice, various pathological aspects under the secondary phase of SCI were examined between WT and $\mathrm{p} 38 \alpha^{+/-}$mice. We finally showed that oral administration of SB239063, a p38 $\alpha$-specific inhibitor might be beneficial to functional recovery after SCI.

\section{MATERIALS AND METHODS}

\section{Animals}

All animal procedures conformed to the Japanese regulations for animal care and use, following guideline for Animal Experimentation of the Japanese Association for Laboratory Animal Science, and were approved by the Animal Care and Use Committee of Chiba University. Male mice heterozygous for targeted disruption of the p38 $\alpha$ gene (Tamura et al., 2000) were crossed with C57BL6J female mice (Tokyo Experimental Animal Co., Tokyo, Japan) to generate $\mathrm{p} 38 \alpha^{+/-}$and $\mathrm{p} 38 \alpha^{+/+}$ [Wild type (WT)] mice. Genotyping by PCR analysis of tailderived DNA was performed according to our previous report (Takanami-Ohnishi et al., 2002).

\section{SCI Model}

Male WT and $\mathrm{p} 38 \alpha^{+/-}$mice aged 10-14 weeks were used for each experiment. Mice were deeply anesthetized with isoflurane. Laminectomy was performed at the thoracic levels of T8-10 to expose the spinal cord (SC), taking care not to damage the SC. Mice of the sham-operated group underwent laminectomy alone. Using a micro dissecting forceps, mice of the SCI group underwent right lateral hemisection at T9. Then, the muscle layer and the skin were sutured. After awaking fully from anesthesia, paralysis of the right hindlimb was assessed. The right hindlimb movement was not observed in this SCI model mice at the surgical day (day 0 ). At day 0 , therefore, mice showing the movement of right hindlimb or with paraplegia were excluded from the following assessment and experiments. The urine was squeezed out by manual abdominal pressure on the bladder twice daily until reflex bladder function would be recovered. To determine the effect of a p38 $\alpha$ inhibitor on SCI, each mouse received oral administration of SB239063 in acidified $0.5 \%$ tragacanth $(10 \mathrm{mg} / \mathrm{kg}$ per day; Sigma-Aldrich, St. Louis, MO, USA) at 1, 2, and $3 \mathrm{dpi}$, and the control group received oral administration of vehicles, acidified $0.5 \%$ tragacanth.

\section{Behavior Study}

We evaluated the motor function of the SCI-associated paralyzed hindlimbs from 0 to 28 days post-injury (dpi), based on Basso Mouse Scale (BMS) (Basso et al., 2006). The BMS is a 9 point scale for assessment of functional recovery of mice's hindlimbs. Mice were forced to walk in an open field, and their right hindlimbs movement was observed for 4 min to score based on BMS.

\section{Tissue Preparation and Histological Analysis}

Mice were anesthetized lethally and transcardially perfused with ice-cold phosphate-buffered saline (PBS). SCs including the lesions were carefully dissected out, fixed overnight in $4 \%$ paraformaldehyde and subsequently immersed in 30\% sucrose for 2 days to cryoprotect the tissues. After embedding into OCT 
compound, the samples were transversely or sagittally sectioned at a thickness of $20 \mu \mathrm{m}$. Sagittal and transverse sections were stained with hematoxylin-eosin ( $\mathrm{HE}$ ) and by a Kluver-Barrera's (KB) method, respectively. The injury-associated leukocyte infiltration area and Luxol Fast Blue (LFB)-staining area in sections stained with $\mathrm{HE}$ and by a $\mathrm{KB}$ method, respectively, were quantified using Macromax MVC-DU (GOKO, Kanagawa, Japan).

\section{Tracing Study}

For anterograde tracing of axons, a total of $2 \mu \mathrm{l}$ of $10 \%$ Texas Redconjugated biotinylated dextran amine (Texas Red-BDA; Vector Laboratories, Burlingame, CA, USA) was injected into four sites of sensorimotor cortex in the left side (contralateral to the side of injured SC) at $14 \mathrm{dpi}$. The scalp was cut and a hole was carefully drilled into the skull, and then Texas Red-BDA was injected into the sensorimotor cortex using a $10 \mu \mathrm{l}$ Hamilton microsyringe. The scalp was closed with suture. Mice applied with Texas Red-BDA were killed at $28 \mathrm{dpi}$. Sagittal sections (20 $\mu \mathrm{m}$ thick) from the SCs were observed by a fluorescence microscope (Axio Imager A2, Zeiss, Oberkochen, Germany). Texas Red-BDA-staining area in a visual field was quantified using ImageJ 1.45 .

\section{Immunofluorescence Study}

The freshly cut sagittal sections (20 $\mu \mathrm{m}$ thick) placed on polyL-lysine-coated slides were pretreated with 1:10 FcR blocking agent (Miltenyi Biotec, Gladbach, Germany) for $10 \mathrm{~min}$ and reacted with various primary antibodies as follows: anti-CNPase (Sigma-Aldrich, St. Louis, MO, USA) to label oligodendrocytes, anti-cleaved caspase-3 (Cell Signaling Tech., Beverly, MA, USA) to label apoptotic cells, Cy3-conjugated anti-glial fibrillary acidic protein (GFAP; Sigma-Aldrich) to label astrocytes, anti-Ibal (WAKO, Osaka, Japan) to label microglia or macrophages, Cy3conjugated anti-NG2 (Merck Millipore, Billerica, MA, USA) to label to oligodendrocyte precursors, FITC-conjugated antiCD45.2 (BioLegend, San Diego, CA, USA) to label leukocytes, biotin-labeled anti-CD3 (Affymetrix, Santa Clara, CA, USA) to label $\mathrm{T}$ lymphocytes. After staining with each appropriate fluorescein-conjugated second antibody or streptavidin, $4^{\prime}$, 6-diamidino-2-phenylindole (DAPI) was applied for nuclear staining before the final washing step. The sections were observed by a fluorescence microscope. In case of counting cells immunoreacted with antibodies, 4-5 sections from each SC were randomly selected. Under $200 \times$ magnification, two fields within $1 \mathrm{~mm}$ centered on the lesion epicenter in the SCI group or the corresponding segment in the sham-operated group were randomly chosen in each section, and fluorescent signalexpressing cells were counted and averaged $\left(/ 0.1 \mathrm{~mm}^{2}\right)$.

\section{Western Blot Array Analysis}

Male WT and $\mathrm{p} 38 \alpha^{+/-}$mice of the sham-operated and SCI groups were anesthetized and sacrificed at $7 \mathrm{dpi}$. Then, each unilateral SC in the right side including with the injured region was dissected out, and cut with the length of $6 \mathrm{~mm}$ from the edge of the rostral lesion to caudal side. The SC sample was homogenized, and centrifuged at $9000 \times g$ for $20 \mathrm{~min}$ at $4^{\circ} \mathrm{C}$.
The resulting supernatant was subjected to protein assay. Protein sample (60 $\mu \mathrm{g} / \mathrm{mouse}$ ) from five mice of each group (WT-sham, WT-SCI, p38 $\alpha^{+/-}$-sham, p38 $\alpha^{+/-}$-SCI) was mixed $(300 \mu \mathrm{g}$ in each group) and subjected to RayBio ${ }^{\circledR}$ Biotin Label-based Mouse Antibody Array 1 (RayBiotech, Norcross, GA, USA), and changes in expression levels of 308 inflammation-related proteins in the samples were evaluated. The array was performed according to the manufacturer's instructions. Using a densitometer, each signal was normalized to the positive internal controls included in the array membrane and expressed as induction ratio of the sham-operated value.

\section{Statistical Analysis}

All analyses were conducted through GraphPad Prism Version 6 (GraphPad Software, San Diego, CA, USA). Statistical significance was determined by Mann-Whitney $U$ test, Student's $t$-test or analysis of variance (ANOVA) followed by Tukey's test, and $P$-value of $<0.05$ were considered to be significant.

\section{RESULTS AND DISCUSSION}

\section{Recovery of Hindlimb Locomotor Behavior Related to Histopathological Findings in $\mathrm{SC}$ after $\mathrm{SCl}$ between p38 $\alpha^{+/-}$and WT Mice}

We first addressed to whether a single copy disruption of $p 38 \alpha$ gene might affect functional recovery of hindlimb after lateral hemisection employed as SCI model in this study. Although such a laceration injury of SC is not typically seen clinically, a hemisection model is suitable to investigate the pathophysiological elements inhibiting or promoting axonal regeneration across or around the laceration injury as well as the resulting functional impairment and potential recovery (Onifer et al., 2007). As shown in Figure 1, p38 $\alpha^{+/-}$mice showed significantly less severe neurological function of paralyzed SCIassociated right hindlimbs at $7 \mathrm{dpi}$, and then the more improved locomotor function in $\mathrm{p} 38 \alpha^{+/-}$mice than in WT mice remained until 28 dpi. Therefore, a single copy disruption of $p 38 \alpha$ gene suppressed the functional disturbance in the hemisection model of SCI.

Then, histopathological changes such as leukocytic infiltration-associated lesion area, myelinated area and axonal regeneration between the two genotypes were examined after SCI (Figure 2). We calculated the leucocytic infiltration area using sagittal sections stained with $\mathrm{HE}$ at 1,2, and 4 wpi (Figures 2A,C). There was no significant difference in the size of leukocyte infiltration-associated lesion between the two genotypes at 1 wpi. The SCI-induced lesion was reduced in a time-dependent manner in the two genotypes at 2 and 4 wpi but significantly smaller in $\mathrm{p} 38 \alpha^{+/-}$mice than in WT mice at each time point. These results suggest that the functional and histopathological recovery after SCI may be enhanced or accelerated in $\mathrm{p} 38 \alpha^{+/-}$mice compared with WT mice. Remyelination of regenerated axons are likely to be one of key mechanisms involved in the spontaneous recovery of motor 


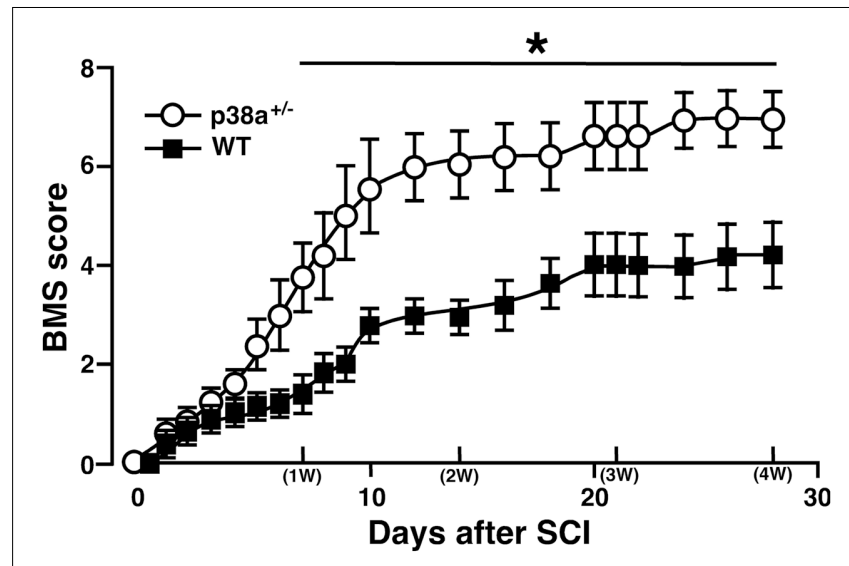

FIGURE 1 | Time course of hindlimb locomotor behavior after spinal cord injury (SCl). Wild type (WT) and $p 38 \alpha^{+/-}$mice with SCl were observed daily until $28 \mathrm{dpi}$, and scored based on BMS. Data are shown as mean \pm SEM ( $n=5)$. The difference between WT (filled squares) and p38 $\alpha^{+/-}$(open circles) mice was statistically significant $\left({ }^{*} P<0.05\right)$ as determined by Mann-Whitney $U$ test for unpaired values at each time point.

function after SCI (Lu et al., 2012). As shown in Figure 2B, Luxol Fast Blue (LFB)-stained area on transverse section of the SC of the two genotypes was significantly smaller in the SCI group compared with the sham-operated group. And, the LFB-positive ratio was significantly larger in $\mathrm{p} 38 \alpha^{+/-}$mice than in WT mice at 4 wpi (Figure 2C). At 2 wpi, the LFB-positive staining in the white matter of SC of the two genotypes was much weaker than the case at 4 wpi (data not shown), suggesting that axonal remyelination may occur at least over a time period ranging from 2 to 4 wpi. Likewise, axons labeled by an anterograde tracer, Texas Red-BDA were more frequently observed in caudal part of the SC of p38 $\alpha^{+/-}$mice than of WT mice at 4 wpi, a semiquantitative analysis of which showed a significant difference between the both genotypes (Figure 2D). The labeled frequency in rostral part of the SC was equally high between the two genotypes because axonal degeneration predominantly occurred in caudal part of the SC, and axons are intact in $5 \mathrm{~mm}$ rostral to the lesion epicenter of SC after the hemi-section injury. These results suggest that axonal regeneration and remyelination after SCI may be enhanced or accelerated in $\mathrm{p} 38 \alpha^{+/-}$mice compared with WT mice. To elucidate the mechanism underlying the improved signs of SCI in p38 $\alpha^{+/-}$mice, we focused on various pathological events at 1 and 2 wpi in which the difference in improvement of spontaneous locomotor ability after SCI between $\mathrm{p} 38 \alpha^{+/-}$mice and WT mice was recognized and then manifested.

\section{Characterization of Inflammatory Response and Evaluation of Oligodendrocyte Apoptosis between p38 $\alpha^{+/-}$and WT Mice at 1 wpi}

A significant importance of leukocytes-mediated inflammatory reaction is well known in the development of SCI. In particular, $\mathrm{T}$ lymphocytes invade the lesion site, concomitantly

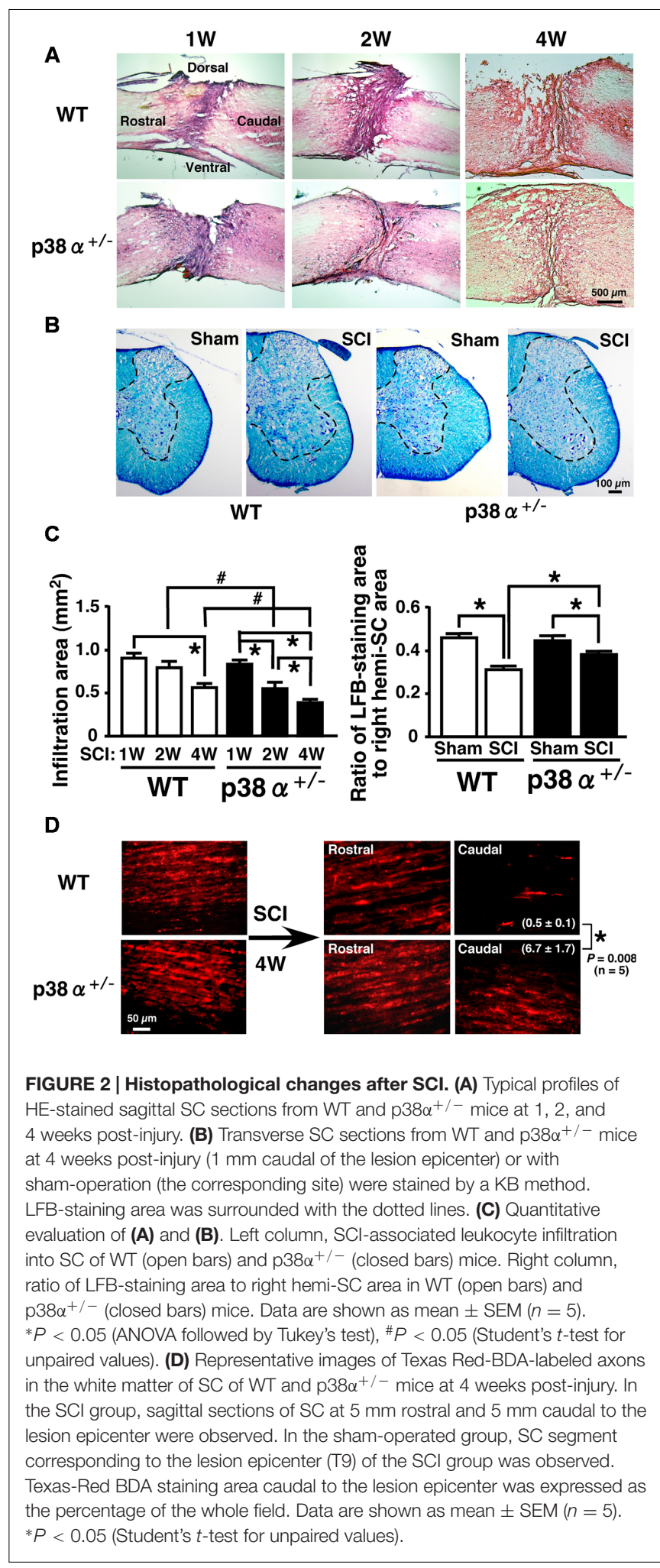

to macrophages and secrete cytokines in the lesion epicenter, which results in axonal damage and motor neuron apoptosis after SCI (Brunn et al., 2008; Beck et al., 2010). Moreover, a previous report demonstrated that the expression of p38 was 
enhanced in resident and infiltrating immune cell after SCI (Stirling et al., 2008). Thus, we elucidated cell populations of leukocytes and $\mathrm{T}$ lymphocytes detected as $\mathrm{CD}_{4} 5^{+}$and $\mathrm{CD}^{+}$, respectively, in the lesion. As shown in Figures 3A,B, CD $45^{+}$ and $\mathrm{CD}^{+}$cells were observed in the lesion of the two genotypes at $1 \mathrm{wpi}$, each number of which was significantly lower in p38 $\alpha^{+/-}$mice than WT mice. In general, neutrophils are a major cell population of $\mathrm{CD} 45^{+}$cells and contribute to both the progression of damage and the tissue repair after SCI (Neirinckx et al., 2014). However, $\mathrm{Ly} 6 \mathrm{G}^{+}$neutrophils were hardly detected in the lesion at 1 wpi (data not shown), which was supported by a previous report that neutrophil recruitment showed fast kinetics reaching a peak at 1 day and immediately declining to the baseline within several days (Donnelly and Popovich, 2008). Considering cell types of leukocytes in the inflammation of SCI, thus, $\mathrm{CD} 45^{+} \mathrm{CD} 3^{-}$cells in the lesion at 1 wpi may be mainly monocytes/macrophages. At the same time point, we also investigated the comprehensive analysis of SCI-induced change in expression of inflammation-related proteins in the SC. As shown in Figure 3C, we found 15 molecules [C-X-C motif chemokine 12 (CXCL12); Eotaxin-2; Galectin-3; insulin-like growth factor 2 (IGF-II), IL-2 receptor $\gamma$ (IL-2R $\gamma$ ); IL-9; IL-9 receptor (IL-9R); IL-12/p70; Kremen-1; macrophage inflammatory protein $1 \alpha$ (MIP-1 $\alpha)$; MIP-2; matrix metalloproteinase 9 (MMP-9); Osteoactivin; tissue inhibitors of metalloproteinase 4 (TIMP-4); Toll-like receptor 2 (TLR2)] showing a clear difference in their expression between WT and p38 $\alpha^{+/-}$mice. The lower expression of MIP-1 $\alpha$, MIP-2, and MMP-9 was corroborated as less leucocyte infiltration in the injured SC of p38 $\alpha^{+/-}$mice (Jaerve and Müller, 2012). In particular, MMP-9 has been thought to open the blood-SC barrier and promote migration of leukocytes into the lesion, which may directly influence the severity of SCI (Noble et al., 2002). It has been also reported that MMP-9 and CXCL12 function synergistically to facilitate migration of blood-borne monocyte (Zhang et al., 2011), although CXCL12 has been regarded as one of key chemoattractants that regulates migration of homeostatic stem and progenitor cells in animal models of CNS injury and promote axonal sprouting (Jaerve and Müller, 2012). Therefore, it can be speculated that the decreased expression of both MMP-9 and CXCL12 in p38 $\alpha^{+/-}$mice may lead to the less infiltrating leucocytes (Stirling et al., 2008). Furthermore, MIP$1 \alpha$ and CXCL12 can recruit T lymphocytes into the injured site (Ousman and David, 2001; Jaerve and Müller, 2012). Thus, their decreased expression may be closely related to the reduction of $\mathrm{T}$ cell infiltration in $\mathrm{p} 38 \alpha^{+/-}$mice. On the other hand, Galectin3 and TLR2 have been reported to have protective effects on SCI through regulating inflammatory response (Kigerl et al., 2007; Stirling et al., 2014; Gensel et al., 2015; Mostacada et al., 2015). Among the 15 molecules, only SCI-induced Galectin3 was higher in $\mathrm{p} 38 \alpha^{+/-}$mice compared with WT mice, suggesting that the increase of Galectin-3 may also contribute to the less infiltrating leucocytes in $\mathrm{p} 38 \alpha^{+/-}$mice. Further study is needed to elucidate whether functional inhibition of each molecule by its neutralizing antibody affects the severity of SCI. However, the decrease in concomitant infiltration of monocytes/macrophages and $\mathrm{T}$ lymphocytes associated with
A
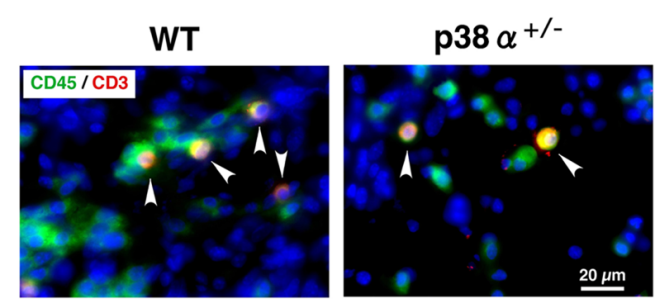

B
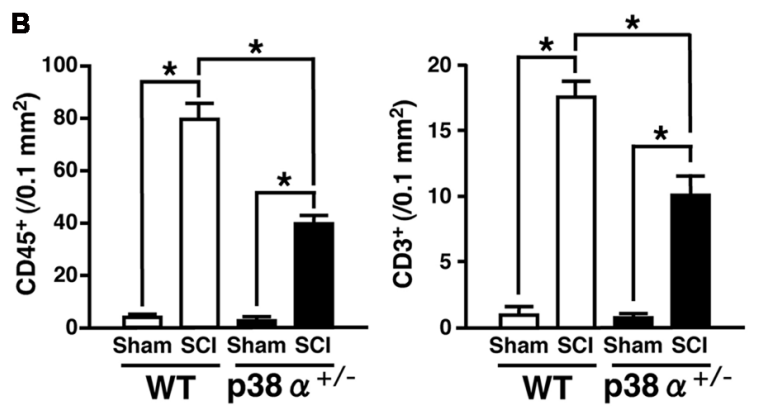

C

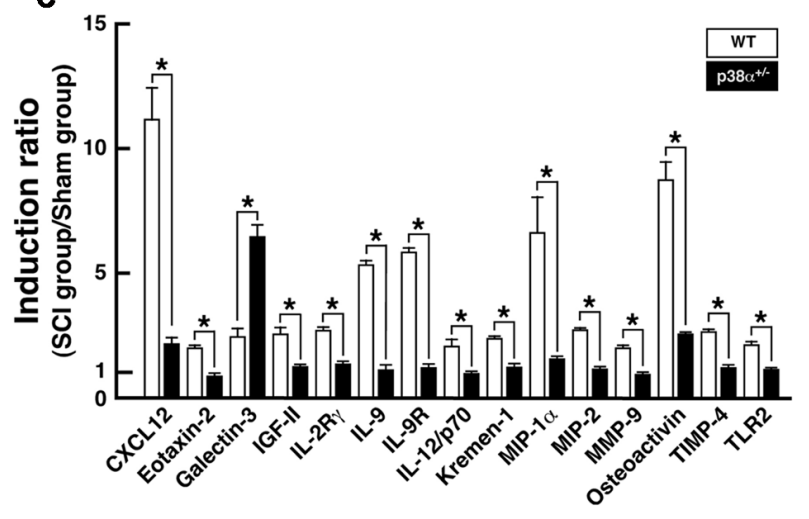

FIGURE 3 | Inflammatory profiles 1 week after SCI. (A) Typical profile of $\mathrm{CD}^{+} \mathrm{CD}_{4} 5^{+}$cells in the lesion epicenter of the two genotypes at 1 week post-injury. Asterisks indicate double-positive cells. (B) Quantitative evaluation of (A). $\mathrm{CD}^{+}$cells and $\mathrm{CD} 45^{+}$cells in the injured site within $500 \mu \mathrm{m}$ rostrocaudal of the lesion epicenter increased in both WT (open bars) and p38 $\alpha^{+/-}$(closed bars) mice at 1 week post-injury, the number of which was significantly lower in $\mathrm{p} 38 \alpha^{+/-}$mice than WT mice. Data are shown as mean \pm SEM $(n=5)$. ${ }^{*} P<0.05$ (ANOVA followed by Tukey's test).

(C) SCl-induced changes in expression of cytokines in the SC between WT and $\mathrm{p} 38 \alpha^{+/-}$mice. Collected SC protein sample from five mice of each group (WT-sham, WT-SCl, p38 $\alpha^{+/-}$-sham or p38 $\alpha^{+/-}$-SCl) were subjected to protein array for 308 molecules. Three independent experiments were conducted (15 mice in each group). Using a densitometer, each signal was normalized to the positive internal controls included in the array membrane (P1-a), and expressed as induction ratio of the sham-operated value. Among 61 molecules ( $\geq 2$, induction ratio in WT group), 15 molecules showed a significant difference in their induction ratio between the two genotypes. Data are shown as mean \pm SEM $(n=3$ ). The difference between WT (open squares) and p38 $\alpha^{+/-}$(filled squares) mice was statistically significant $\left({ }^{*} P<0.05\right)$ as determined by Student's $t$-test for unpaired values.

the changes in expression of several cytokines/chemokines may contribute to the less development of SCI in $\mathrm{p} 38 \alpha^{+/-}$ mice.

In CNS, p38 mainly localizes in myelin sheath but not in axon (Maruyama et al., 2000). It has been reported that inhibition 
of p38 prevents myelin structure destruction associated with oligodendrocytic apoptosis and ameliorates neurological deficits after SCI (Horiuchi et al., 2003). It has also been reported that apoptosis of oligodendrocyte would occur about 1 week after SCI and cause demyelination and axonal disturbance (Li et al., 1999; Dong et al., 2003). As a reliable strategy, colocalized cells of CNPase and cleaved caspase- 3 are regarded as oligodendrocytes under programmed cell death in human SCI (Emery et al., 1998). Then, we evaluated a cell population of cleaved caspase- $3^{+} \mathrm{CNPase}^{+}$cells in the white matter of the lesion. As shown in Figure 4, cleaved caspase- $3^{+} \mathrm{CNPase}^{+}$cells were typically observed in the SCI group of the two genotypes at 1 wpi (A), the number of which was significantly lower in $\mathrm{p} 38 \alpha^{+/-}$mice than WT mice (B). On the other hand, $\mathrm{Nissle}^{+}$neuronal cells decreased in the gray matter of SC around the lesion epicenter in the two genotypes at 1 wpi, which was significantly moderate in $\mathrm{p} 38 \alpha^{+/-}$mice than WT mice (Supplementary Figure 1). These results indicated that $\mathrm{p} 38 \alpha^{+/-}$ mice showed resistance to cell death of oligodendrocytes and neurons at 1 wpi. Hence, the inflammatory responses and cell death of oligodendrocytes and neurons after SCI were reduced by a single copy disruption of $p 38 \alpha$ gene. Next, we elucidated a role of $\mathrm{p} 38 \alpha$ in the tissue regeneration process after SCI.

\section{Astrocytic Scar Formation and Increase in Oligodendrocyte Precursor Cells after $\mathrm{SCl}$ between p38 $\alpha^{+/-}$and WT Mice}

In a great numbers of studies, glial scar by reactive astrocytes has been regarded as physical barriers to successful axon regeneration (Silver and Miller, 2004). In contrast, recent studies have provided the possibility that reactive astrogliosis have beneficial effects in axonal regeneration via forming the astrocyte bridge, a scaffold for axonal growth (Anderson et al., 2016; Mokalled et al., 2016). On the other hand, activated microglias and macrophages markedly infiltrate into the lesion of CNS injury, the spread degree of which is closely associated with substantial tissue repair and functional restoration (Penkowa et al., 1999). Thus, the cellular formation of $\mathrm{GFAP}^{+}$astrocytes and $\mathrm{Iba}^{+}{ }^{+}$microglias/macrophages between p38 $\alpha^{+/-}$mice and WT mice was examined at 2 wpi. As shown in Figure 5, astrocytic scar formation was observed in the epicenter of the two genotypes, which was more massive in WT mice ( $\mathrm{B}$ and $\mathrm{C}$ ) than $\mathrm{p} 38 \alpha^{+/-}$mice ( $\mathrm{F}$ and $\mathrm{G})$. Likewise, a larger numbers of reactive astrocytes with hypertrophied somas and long processes were observed in WT mice (D) than $\mathrm{p} 38 \alpha^{+/-}$mice $(\mathrm{H})$. These phenomena were supported by a previous study that astrogliosis after CNS damage might be attenuated in astrocyte-specific p $38 \alpha$-knockout mice (Roy Choudhury et al., 2014). On the other hand, $\mathrm{Iba1}^{+}$ microglias/macrophages were accumulated more compactly between rostrocaudal $\mathrm{GFAP}^{+}$reactive astrocytes in $\mathrm{p} 38 \alpha^{+/-}$ mice than WT mice (Figures 5B,C,F,G). Likewise, the distance between rostrocaudal glial scars was shorter in $\mathrm{p} 38 \alpha^{+/-}$mice than WT mice (Figures 5A,E), indicating that contraction of lesion area was enhanced in $\mathrm{p} 38 \alpha^{+/-}$mice compared with WT

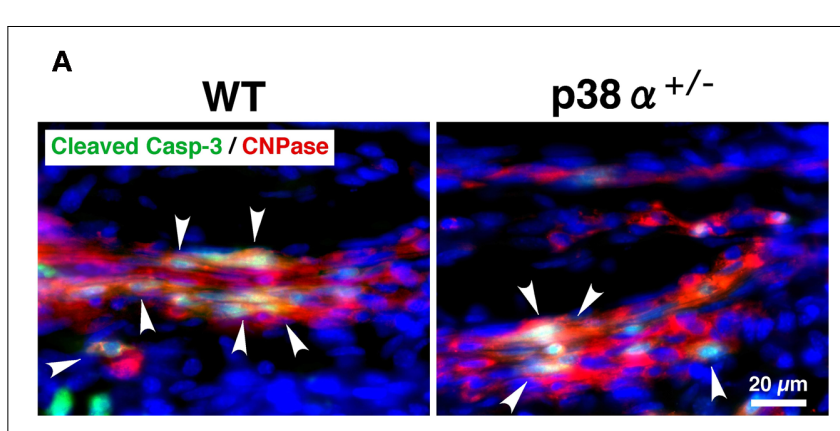

B

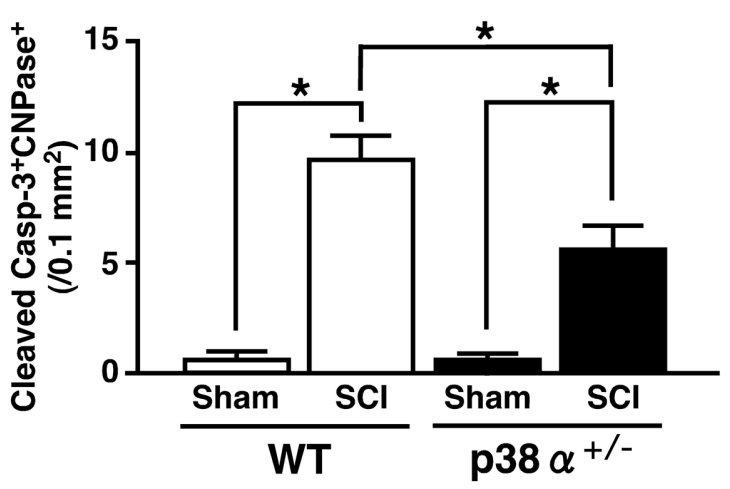

FIGURE 4 | Changes in numbers of apoptotic oligodendrocytes in the white matter 1 week after SCI. (A) Typical profile of cleaved caspase- $3^{+}$CNPase ${ }^{+}$cells in the white matter at the caudal boarder of the lesion of the two genotypes at 1 week post-injury. Asterisks indicate double-positive cells. (B) Cleaved caspase- $3^{+} \mathrm{CNPase}^{+}$cells in the injured site within $500 \mu \mathrm{m}$ rostrocaudal of the lesion epicenter increased in both WT (open bars) and p38 $\alpha^{+/-}$(closed bars) mice at 1 week post-injury, the number of which was significantly lower in p38 $\alpha^{+/-}$mice than WT mice. Data are shown as mean \pm SEM $(n=4-5) . * P<0.05$ (ANOVA followed by Tukey's test).

mice. It has been reported that the Stat3-upregulated migratory activity of reactive astrocytes to seclude inflammatory cells enhances contraction of lesion area and functional restoration after SCI (Okada et al., 2006). Moreover, a loss of function of p38 $\alpha$ in astrocyte negatively affects its cellular migration activity (Roy Choudhury et al., 2014). Thus, more typical compaction of microglias/macrophages associated with smaller wound area in $\mathrm{p} 38 \alpha^{+/-}$mice compared with $\mathrm{WT}$ mice is of interest. Probably, at least the less infiltration of inflammatory cells related to expression of proinflammatory cytokines (Figure 3) may positively affect the wound healing process in $\mathrm{p} 38 \alpha^{+/-}$ mice even though astrogliosis-forming activity is moderate compared with WT mice. In addition, it was also provided the possibility that the contribution of NSCs to wound healing/tissue regeneration process after SCI might be potentiated in $\mathrm{p} 38 \alpha^{+/-}$ mice.

Multipotent NSCs are defined as cells that can self-renew and differentiate into the three neuronal lineages, neuron, astrocyte, and oligodendrocyte (Gage, 2000). In the SC, NSCs known as ependymal cells in the central canal proliferate rapidly after SCI and differentiate into more than half the astrocytes in the 

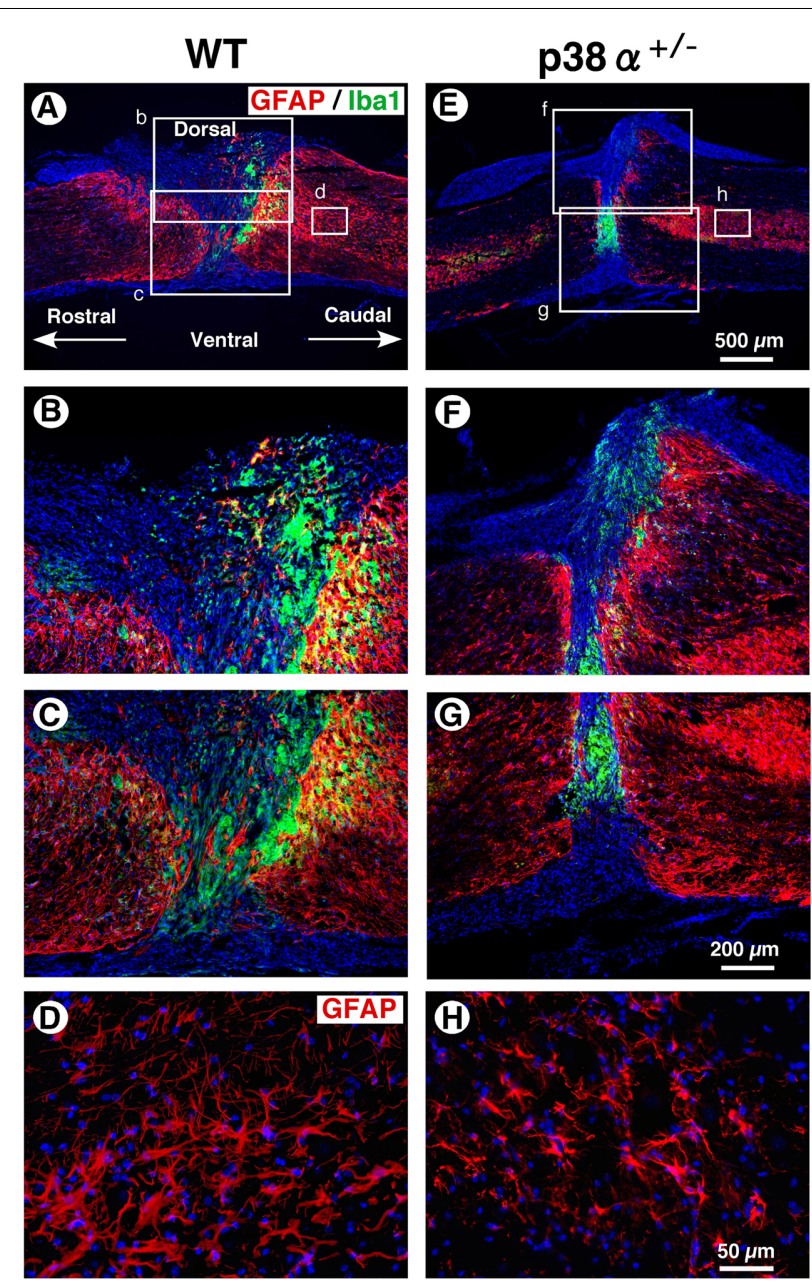

FIGURE 5 | Astrocytic scar formation and

neuroinflammation-associated cells at 2 weeks post-injury. (A,E) Representative images of sagittal sections showing $\mathrm{GFAP}^{+}$reactive astrocytes and $\mathrm{lba}{ }^{+}$cells at 2 weeks post-injury. (B,C,F,G) Higher magnification images from the boxed area, b, c, f and $g$ in $(\mathbf{A})$ and $(\mathbf{E})$. Iba1 ${ }^{+}$cells were compacted to the lesion center between rostrocaudal reactive astrocytes, which was more apparent in the SC of p38 $\alpha^{+/-}$mice than that of WT mice. (D,H) Higher magnification images of GFAP $^{+}$reactive astrocytes from the boxed area, $d$ (in A) and $\mathrm{h}$ (in E). GFAP ${ }^{+}$reactive astrocytes at $1 \mathrm{~mm}$ caudal to the lesion epicenter decreased in $\mathrm{p} 38 \alpha^{+/-}$mice compared with WT mice.

glial scar and a small amount of oligodendrocytes (BarnabéHeider et al., 2010). Likewise, in vitro, NSCs derived from adult WT mice easily and exclusively differentiate into astrocyte with repeating cell passages without appropriate neurotrophic factors (Seaberg and van der Kooy, 2002; Bull and Bartlett, 2005). Hence, the low differentiation capacity of adult NSC for neural and oligodendrocytic lineages can limit the recovery from SCI. We previously reported that NSCs in the adult hippocampus of $\mathrm{p} 38 \alpha^{+/-}$mice have much higher self-renewal activity and neural differentiation capacity compared with those of WT mice (Yoshioka et al., 2015). These findings tempted us to think that activity of progenitor cells for oligodendrocyte and neuron might be upregulated in $\mathrm{p} 38 \alpha^{+/-}$mice under the tissue regeneration process after SCI. To elucidate this point, we observed cell populations of oligodendrocyte precursor cells (OPCs) and neural progenitor cells in the lesion epicenter of $\mathrm{SC}$ at 2 wpi. As we expected, the number of $\mathrm{NG}^{+}$OPCs in the white matter of the lesion increased in the two genotypes at 2 wpi, which was significantly higher in $\mathrm{p} 38 \alpha^{+/-}$mice than in WT mice (Figure 6). NG2 ${ }^{+}$OPCs into the injured SC can enhance remyelination of spared axons and improve functional recovery after SCI (Whittaker et al., 2012). Thus, in conjunction with the results shown in Figures $\mathbf{2 B}, \mathbf{C}$, the increase in OPCs in $\mathrm{p} 38 \alpha^{+/-}$mice after SCI may contribute to the efficiency of remyelination under the tissue regeneration process. We also determined that $\mathrm{NG}^{+}$cells were more accumulated along the laceration/epicenter in $\mathrm{p} 38 \alpha^{+/-}$mice compared with WT mice (data not shown). This accumulation was very similar to typical histopathological findings in CNS damages including SCI (Tan et al., 2005). NG2 is a member of chondroitin sulphate proteoglycans (CSPGs) generally known to be repulsive to growing axons. However, NG2 also called CSPG4 has been recently recognized as a promoting molecule for axonal growth and regeneration (Yang et al., 2006). In fact, it has been demonstrated that astrocytes forming a bridge across a scar after SCI highly express CSPG4/NG2 (Anderson et al., 2016). Likewise, $\mathrm{NG}^{+}$OPCs provide an adhesive substrate for axonal growth by forming a bridge after SCI (Busch et al., 2010). Although we could not detected the astrocytic bridge between rostrocaudal glial scars in the two genotypes (Figure 5), the fact that accumulation of $\mathrm{NG}^{+}$OPCs along the laceration/epicenter was more apparent in $\mathrm{p} 38 \alpha^{+/-}$mice compared with WT mice might affect the efficiency of axonal regeneration between the two genotypes. In addition to the upregulation of OPC recruitment in $\mathrm{p} 38 \alpha^{+/-}$mice, an increase in $\mathrm{DCX}^{+}$neural progenitor cells in the gray matter of the lesion was bigger in $\mathrm{p} 38 \alpha^{+/-}$mice than in WT mice at 2 wpi. Some of the $\mathrm{DCX}^{+}$neural progenitor cells also expressed Nestin and were observed in area proximal to the lesion epicenter in the two genotypes. Notably, $\mathrm{DCX}^{+} \mathrm{Nestin}^{+}$cells were also detected within the epicenter in case of $\mathrm{p} 38 \alpha^{+/-}$ mice but not WT mice (Supplementary Figure 2). DCX ${ }^{+} \mathrm{Nestin}^{+}$ cells have been identified as resident multipotent NSCs in the SC meninges and at least contribute to parenchymal reaction following SCI (Decimo et al., 2011). Although it is still unclear whether the $\mathrm{DCX}^{+} \mathrm{Nestin}^{+}$cells recruited into the lesion contribute to neural regeneration, the finding that recruitment of neuroblasts and resident NSCs to the lesion was upregulated in $\mathrm{p} 38 \alpha^{+/-}$mice may affect the subsequent tissue regeneration in concert with the enhanced recruitment of OPCs.

Together, a single copy disruption of $p 38 \alpha$ gene affected the tissue degeneration and regeneration processes and improved the functional recovery from SCI. However, the question arose as to whether the SCI-augmented p38 activation was inhibited in the SC of p38 $\alpha^{+/-}$mice. Although SCI did not affect each expression level of $\mathrm{p} 38 \alpha$ in the two genotypes, the SCI-augmented p38 activation was less in $\mathrm{p} 38 \alpha^{+/-}$mice compared with WT mice (Supplementary Figure 3). Therefore, we finally evaluated the effect of a p38 inhibitor on the functional recovery from SCI. 


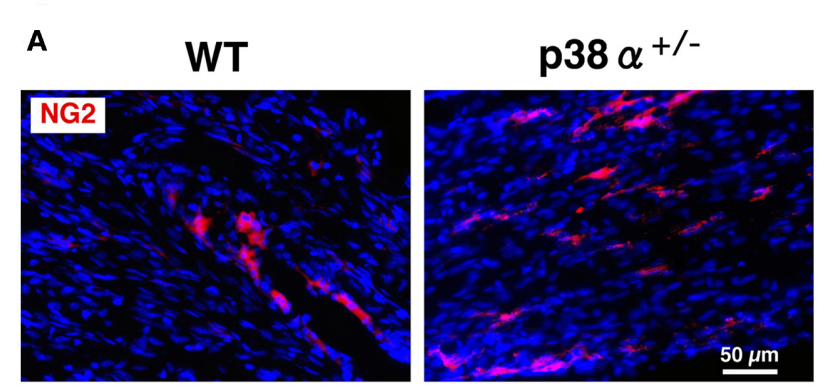

B

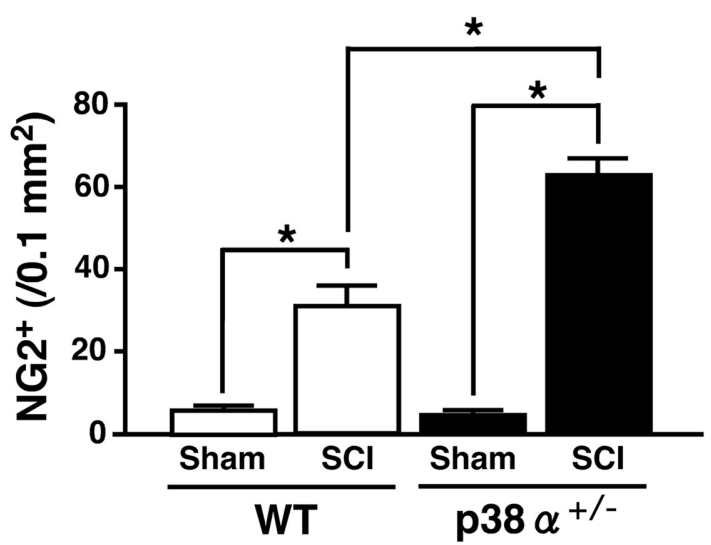

FIGURE 6 | Change in numbers of $\mathrm{NG2}^{+}$cells in the white matter at 2 weeks after $\mathbf{S C l}$. (A) Typical profile of $\mathrm{NG}^{+}$cells in the lesion epicenter of the two genotypes at 2 weeks post-injury. (B) NG2 ${ }^{+}$cells in the injured site within $500 \mu \mathrm{m}$ rostrocaudal of the lesion epicenter in the SCl group were increased in the two genotypes at 1 week post-injury, the number of which was significantly higher in $\mathrm{p} 38 \alpha^{+/}-$mice (closed bars) than WT mice (open bars). Data are shown as mean $\pm \operatorname{SEM}(n=4-5) . * P<0.05$ (ANOVA followed by Tukey's test).

\section{A p38 Inhibitor Improved Locomotor Recovery after SCI}

Unlike the previous studies on the association between SCI and a p38 inhibitor (Horiuchi et al., 2003; Stirling et al., 2008), we used SB239063 as a p38 inhibitor that is an orally active and transferable across the blood-cerebrospinal fluid barrier. In the preliminary study, we confirmed that an oral administration of SB239063 (10 mg/kg body weight) could efficiently inhibit the SCI-induced p38 $\alpha$ activity (Supplementary Figure 4). Then, based on a previous report that the strong activation of p38 MAPK in the injured SC increases from $12 \mathrm{~h}$ to 3 days (Song et al., 2013), we orally administrated SB239063 to WT mice $(10 \mathrm{mg} / \mathrm{kg}$ per day) at 1, 2, and $3 \mathrm{dpi}$. As we expected, the BMS score was significantly higher in the SB239063-treated group compared with the vehicle group over a time period ranging from 6 to $28 \mathrm{dpi}$ (Figure 7), indicating that a pharmacological inhibition of $\mathrm{p} 38 \alpha$ also improved the recovery of hindlimb behavior after SCI.

Our present result that SB239063 is effective on the functional recovery after lateral hemisection of the SC can be supported by the previous study employing a mild contusion model of SCI by Horiuchi et al. (2003). On the other hand, another study

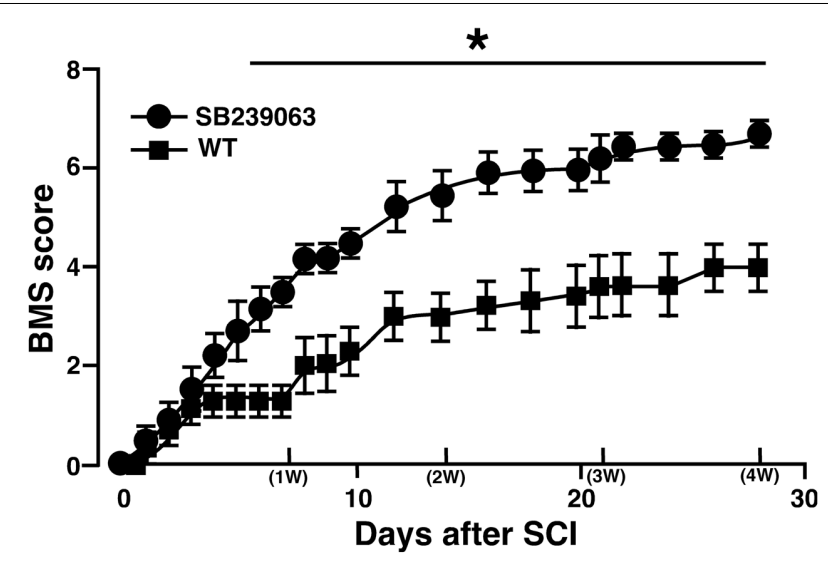

FIGURE 7 | Effect of SB239063 on hindlimb locomotor behavior after SCI. WT mice with $\mathrm{SCl}$ received oral administration of vehicles (filled squares) and SB239063 (10 mg/kg per day, filled circles) at 1, 2, and 3 dpi. Functional recovery from SCl was better in mice with SB239063 treatment than those with vehicle administration. Data are shown as mean $\pm \operatorname{SEM}(n=6)$.

${ }^{*} P<0.05$, a significant difference between the two groups by Mann-Whitney $U$ test for unpaired values at each time point.

demonstrated that a $\mathrm{p} 38 \alpha$ inhibitor failed to improve functional outcome after SCI with moderate contusion (Stirling et al., 2008). Currently, the discrepancy in efficacy of a p38 $\alpha$ inhibitor for SCI between them is difficult to explain. Regardless of the type of SCI model, however, the intensity of SC damage may influence the beneficial effect of a p38 $\alpha$ inhibitor. In addition, the route and schedule of administration with a p38 $\alpha$ inhibitor also affect its efficacy. As a next step, to investigate whether our protocol of SB239063 administration is effective in a moderate contusion model of SCI is needed.

\section{CONCLUSION}

A single copy disruption of $p 38 \alpha$ gene inhibited the tissue degenerative events such as leukocytic infiltration, expression of cytokines/chemokines and apoptosis of oligodendrocyte and neuron and enhanced the tissue regenerative events such as compaction of microglias/macrophages, recruitment of OPCs and NSC and axonal regrowth and remyelination, which resulted in a better functional recovery from SCI. We also demonstrated that a pharmacological inhibition of $\mathrm{p} 38 \alpha$ could recapitulate the better functional recovery from SCI observed in $\mathrm{p} 38 \alpha^{+/-}$ mice. Our present study clearly suggests that $\mathrm{p} 38 \alpha$ contributes to the pathogenesis of SCI and propose that an orally active p38 $\alpha$ inhibitor, SB239063 may have therapeutic benefits for the treatment of SCI.

\section{AUTHOR CONTRIBUTIONS}

HU, KoT, YK developed the concept and designed the experiments. HU, YN, KeT, and YK performed experiments. $\mathrm{KY}$ and KS performed statistical analysis. TS, MHag, and MHat 
gave conceptual advice. HU and YK wrote the paper. All authors discussed the results and implications and commented on the manuscript at all stages.

\section{ACKNOWLEDGMENTS}

This work was supported in part by Grants-in-Aid for Scientific Research ((B), 24390137 to YK) and Young Scientists ((B),

\section{REFERENCES}

Anderson, M. A., Burda, J. E., Ren, Y., Ao, Y., O’Shea, T. M., Kawaguchi, R., et al. (2016). Astrocyte scar formation aids central nervous system axon regeneration. Nature 532, 195-200. doi: 10.1038/nature17623

Barnabé-Heider, F., Göritz, C., Sabelström, H., Takebayashi, H., Pfrieger, F. W., Meletis, K., et al. (2010). Origin of new glial cells in intact and injured adult spinal cord. Cell Stem Cell 7, 470-482. doi: 10.1016/j.stem.2010.07.014

Basso, D. M., Fisher, L. C., Anderson, A. J., Jakeman, L. B., McTigue, D. M., and Popovich, P. G. (2006). Basso Mouse Scale for locomotion detects differences in recovery after spinal cord injury in five common mouse strains. J. Neurotrauma 23, 635-659. doi: 10.1089/neu.2006.23.635

Beck, K. D., Nguyen, H. X., Galvan, M. D., Salazar, D. L., Woodruff, T. M., and Anderson, A. J. (2010). Quantitative analysis of cellular inflammation after traumatic spinal cord injury: evidence for a multiphasic inflammatory response in the acute to chronic environment. Brain 133, 433-447. doi: 10.1093/brain/ awp322

Becker, D., Sadowsky, C. L., and McDonald, J. W. (2003). Restoring function after spinal cord injury. Neurologist 9, 1-15. doi: 10.1097/01.nrl.0000038587. 58012.05

Brunn, A., Utermöhlen, O., Carstov, M., Ruiz, M. S., Miletic, H., Schlüter, D., et al. (2008). CD4 T cells mediate axonal damage and spinal cord motor neuron apoptosis in murine p0106-125-induced experimental autoimmune neuritis. Am. J. Pathol 173, 93-105. doi: 10.2353/ajpath.2008.071101

Bull, N. D., and Bartlett, P. F. (2005). The adult mouse hippocampal progenitor is neurogenic but not a stem cell. J. Neurosci. 25, 10815-10821. doi: 10.1523/ JNEUROSCI.3249-05.2005

Busch, S. A., Horn, K. P., Cuascut, F. X., Hawthorne, A. L., Bai, L., Miller, R. H., et al. (2010). Adult NG2 ${ }^{+}$cells are permissive to neurite outgrowth and stabilize sensory axons during macrophage-induced axonal dieback after spinal cord injury. J. Neurosci. 30, 255-265. doi: 10.1523/JNEUROSCI.3705-09.2010

Decimo, I., Bifari, F., Rodriguez, F. J., Malpeli, G., Dolci, S., Lavarini, V., et al. (2011). Nestin- and doublecortin-positive cells reside in adult spinal cord meninges and participate in injury-induced parenchymal reaction. Stem Cells 29, 2062-2076. doi: 10.1002/stem.766

Dong, H., Fazzaro, A., Xiang, C., Korsmeyer, S. J., Jacquin, M. F., and McDonald, J. W. (2003). Enhanced oligodendrocyte survival after spinal cord injury in Baxdeficient mice and mice with delayed Wallerian degeneration. J. Neurosci. 23, 8682-8691.

Donnelly, D. J., and Popovich, P. G. (2008). Inflammation and its role in neuroprotection, axonal regeneration and functional recovery after spinal cord injury. Exp. Neurol. 209, 378-388. doi: 10.1016/j.expneurol.2007.06.009

Emery, E., Aldana, P., Bunge, M. B., Puckett, W., Srinivasan, A., Keane, R. W., et al. (1998). Apoptosis after traumatic human spinal cord injury. J. Neurosurg. 89, 911-920. doi: 10.3171/jns.1998.89.6.0911

Gage, F. H. (2000). Mammalian neural stem cells. Science 287, 1433-1438. doi: $10.1126 /$ science.287.5457.1433

Gensel, J. C., Wang, Y., Guan, Z., Beckwith, K. A., Braun, K. J., Wei, P., et al. (2015). Toll-like Receptors and Dectin-1, a C-Type Lectin Receptor, Trigger Divergent Functions in CNS Macrophages. J. Neurosci. 35, 9966-9976. doi: 10.1523/JNEUROSCI.0337-15.2015

Horiuchi, H., Ogata, T., Morino, T., Chuai, M., and Yamamoto, H. (2003). Continuous intrathecal infusion of SB203580, a selective inhibitor of p38 mitogen-activated protein kinase, reduces the damage of hind-limb function
93001886 to KY) from the Ministry of Education, Science, Sports and Culture of Japan.

\section{SUPPLEMENTARY MATERIAL}

The Supplementary Material for this article can be found online at: http://journal.frontiersin.org/article/10.3389/fphar. 2017.00072/full\#supplementary-material

after thoracic spinal cord injury in rat. Neurosci. Res. 47, 209-217. doi: 10.1016/ S0168-0102(03)00216-5

Horner, P. J., and Gage, F. H. (2000). Regenerating the damaged central nervous system. Nature 407, 963-970. doi: 10.1038/35039559

Jaerve, A., and Müller, H. W. (2012). Chemokines in CNS injury and repair. Cell Tissue Res. 349, 229-248. doi: 10.1007/s00441-012-1427-3

Kang, Y. J., Chen, J., Otsuka, M., Mols, J., Ren, S., Wang, Y., et al. (2008). Macrophage deletion of p38alpha partially impairs lipopolysaccharide-induced cellular activation. J. Immunol. 180, 5075-5082. doi: 10.4049/jimmunol.180.7. 5075

Kigerl, K. A., Lai, W., Rivest, S., Hart, R. P., Satoskar, A. R., and Popovich, P. G. (2007). Toll-like receptor (TLR)-2 and TLR-4 regulate inflammation, gliosis, and myelin sparing after spinal cord injury. J. Neurochem. 102, 37-50. doi: 10.1111/j.1471-4159.2007.04524.x

Kumar, S., Boehm, J., and Lee, J. C. (2003). p38 MAP kinases: key signalling molecules as therapeutic targets for inflammatory diseases. Nat. Rev. Drug Discov. 2, 717-726. doi: 10.1038/nrd1177

Li, G. L., Farooque, M., Holtz, A., and Olsson, Y. (1999). Apoptosis of oligodendrocytes occurs for long distances away from the primary injury after compression trauma to rat spinal cord. Acta Neuropathol. 98, 473-480. doi: $10.1007 / \mathrm{s} 004010051112$

Lu, P., Blesch, A., Graham, L., Wang, Y., Samara, R., Banos, K., et al. (2012). Motor axonal regeneration after partial and complete spinal cord transection. J. Neurosci. 32, 8208-8218. doi: 10.1523/JNEUROSCI.030812.2012

Maruyama, M., Sudo, T., Kasuya, Y., Shiga, T., Hu, B., and Osada, H. (2000). Immunolocalization of p38 MAP kinase in mouse brain. Brain Res. 887, 350-358. doi: 10.1016/S0006-8993(00)03063-8

Matsuo, Y., Amano, S., Furuya, M., Namiki, K., Sakurai, K., Nishiyama, M., et al. (2006). Involvement of p38alpha mitogen-activated protein kinase in lung metastasis of tumor cells. J. Biol. Chem. 281, 36767-36775. doi: 10.1074/jbc. M604371200

Mokalled, M. H., Patra, C., Dickson, A. L., Endo, T., Stainier, D. Y., and Poss, K. D. (2016). Injury-induced ctgfa directs glial bridging and spinal cord regeneration in zebrafish. Science 354, 630-634. doi: 10.1126/science. aaf 2679

Mostacada, K., Oliveira, F. L., Villa-Verde, D. M., and Martinez, A. M. (2015). Lack of galectin-3 improves the functional outcome and tissue sparing by modulating inflammatory response after a compressive spinal cord injury. Exp. Neurol. 271, 390-400. doi: 10.1016/j.expneurol.2015.07.006

Namiki, K., Matsunaga, H., Yoshioka, K., Tanaka, K., Murata, K., Ishida, J., et al. (2012). Mechanism for p38 $\alpha$-mediated experimental autoimmune encephalomyelitis. J. Biol. Chem. 287, 24228-24238. doi: 10.1074/jbc.M111. 338541

Namiki, K., Nakamura, A., Furuya, M., Mizuhashi, S., Matsuo, Y., Tokuhara, N., et al. (2007). Involvement of p38alpha in kainate-induced seizure and neuronal cell damage. J. Recept. Signal Transduct. Res. 27, 99-111. doi: 10.1080/ 1079989070135785

Neirinckx, V., Coste, C., Franzen, R., Gothot, A., Rogister, B., and Wislet, S. (2014). Neutrophil contribution to spinal cord injury and repair. J Neuroinflammation 11, 150. doi: 10.1186/s12974-014-0150-2

Noble, L. J., Donovan, F., Igarashi, T., Goussev, S., and Werb, Z. (2002). Matrix metalloproteinases limit functional recovery after spinal cord injury by modulation of early vascular events. J. Neurosci. 22, 7526-7535. 
Noubade, R., Krementsov, D. N., Del Rio, R., Thornton, T., Nagaleekar, V., Saligrama, N., et al. (2011). Activation of p38 MAPK in CD4 T cells controls IL-17 production and autoimmune encephalomyelitis. Blood 118, 3290-3300. doi: 10.1182/blood-2011-02-336552

Okada, S., Nakamura, M., Katoh, H., Miyao, T., Shimazaki, T., Ishii, K., et al. (2006). Conditional ablation of Stat 3 or Socs 3 discloses a dual role for reactive astrocytes after spinal cord injury. Nat. Med. 12, 829-834. doi: 10.1038/nm1425

O’Keefe, S. J., Mudgett, J. S., Cupo, S., Parsons, J. N., Chartrain, N. A., Fitzgerald, C., et al. (2007). Chemical genetics define the roles of p38alpha and p38beta in acute and chronic inflammation. J. Biol. Chem. 282, 34663-34671. doi: 10.1074/jbc. M704236200

Onifer, S. M., Rabchevsky, A. G., and Scheff, S. W. (2007). Rat models of traumatic spinal cord injury to assess motor recovery. ILAR J. 48, 385-395. doi: 10.1093/ ilar.48.4.385

Ousman, S. S., and David, S. (2001). MIP-1alpha, MCP-1, GM-CSF, and TNF-alpha control the immune cell response that mediates rapid phagocytosis of myelin from the adult mouse spinal cord. J. Neurosci. 21, 4649-4656.

Oyinbo, C. A. (2011). Secondary injury mechanisms in traumatic spinal cord injury: a nugget of this multiply cascade. Acta Neurobiol. Exp. 71, 281-299.

Penkowa, M., Carrasco, J., Giralt, M., Moos, T., and Hidalgo, J. (1999). CNS wound healing is severely depressed in metallothionein I- and II-deficient mice. J. Neurosci. 19, 2535-2545.

Risco, A., del Fresno, C., Mambol, A., Alsina-Beauchamp, D., MacKenzie, K. F., Yang, H. T., et al. (2012). p38 $\gamma$ and p38 kinases regulate the Toll-like receptor 4 (TLR4)-induced cytokine production by controlling ERK1/2 protein kinase pathway activation. Proc. Natl. Acad. Sci. U.S.A. 109, 11200-11205. doi: 10. 1073/pnas.1207290109

Roy Choudhury, G., Ryou, M. G., Poteet, E., Wen, Y., He, R., Sun, F., et al. (2014). Involvement of p38 MAPK in reactive astrogliosis induced by ischemic stroke. Brain Res. 1551, 45-58. doi: 10.1016/j.brainres.2014.01.013

Seaberg, R. M., and van der Kooy, D. (2002). Adult rodent neurogenic regions: the ventricular subependyma contains neural stem cells, but the dentate gyrus contains restricted progenitors. J. Neurosci. 22, 1784-1793.

Silver, J., and Miller, J. H. (2004). Regeneration beyond the glial scar. Nat. Rev. Neurosci. 5, 146-156. doi: 10.1038/nrn1326

Song, Y., Liu, J., Zhang, F., Zhang, J., Shi, T., and Zeng, Z. (2013). Antioxidant effect of quercetin against acute spinal cord injury in rats and its correlation with the p38MAPK/iNOS signaling pathway. Life Sci. 92, 1215-1221. doi: 10.1016/j.lfs. 2013.05.007

Stirling, D. P., Cummins, K., Mishra, M., Teo, W., Yong, V. W., and Stys, P. (2014). Toll-like receptor 2-mediated alternative activation of microglia is protective after spinal cord injury. Brain. 137, 707-723. doi: 10.1093/brain/awt341

Stirling, D. P., Liu, J., Plunet, W., Steeves, J. D., and Tetzlaff, W. (2008). SB203580, a p38 mitogen-activated protein kinase inhibitor, fails to improve functional outcome following a moderate spinal cord injury in rat. Neuroscience 155, 128-137. doi: 10.1016/j.neuroscience.2008.05.007

Takanami-Ohnishi, Y., Amano, S., Kimura, S., Asada, S., Utani, A., Maruyama, M., et al. (2002). Essential role of p38 Mitogen-activated protein kinase in contact hypersensitivity. J. Biol. Chem. 277, 37896-37903. doi: 10.1074/jbc.M207326200

Tamura, K., Sudo, T., Senftleben, U., Dadak, A. M., Johnson, R., and Karin, M. (2000). Requirement for p38alpha in erythropoietin expression: a role for stress kinases in erythropoiesis. Cell 102, 221-231. doi: 10.1016/S0092-8674(00) 00027-1

Tan, A. M., Zhang, W., and Levine, J. M. (2005). NG2: a component of the glial scar that inhibits axon growth. J. Anat. 207, 717-725. doi: 10.1111/j.1469-7580. 2005.00452.x

Ventura, J. J., Tenbaum, S., Perdiguero, E., Huth, M., Guerra, C., Barbacid, M., et al. (2007). p38alpha MAP kinase is essential in lung stem and progenitor cell proliferation and differentiation. Nat. Genet. 39, 750-758. doi: 10.1038/ng2037

Whittaker, M. T., Zai, L. J., Lee, H. J., Pajoohesh-Ganji, A., Wu, J., Sharp, A., et al. (2012). GGF2 (Nrg1- $\beta 3$ ) treatment enhances $\mathrm{NG}^{+}$cell response and improves functional recovery after spinal cord injury. Glia 60, 281-294. doi: 10.1002/glia.21262

Yang, Z., Suzuki, R., Daniels, S. B., Brunquell, C. B., Sala, C. J., and Nishiyama, A. (2006). NG2 glial cells provide a favorable substrate for growing axons. J. Neurosci. 26, 3829-3839. doi: 10.1523/JNEUROSCI.4247-05.2006

Yoshioka, K., Namiki, K., Sudo, T., and Kasuya, Y. (2015). p38 $\alpha$ controls selfrenewal and fate decision of neurosphere-forming cells in adult hippocampus. FEBS Open Bio 5, 437-444. doi: 10.1016/j.fob.2015.05.001

Zhang, H., Trivedi, A., Lee, J. U., Lohela, M., Lee, S. M., Fandel, T. M., et al. (2011). Matrix metalloproteinase- 9 and stromal cell-derived factor- 1 act synergistically to support migration of blood-borne monocytes into the injured spinal cord. J. Neurosci. 31, 15894-15903. doi: 10.1523/JNEUROSCI.3943-11.2011

Zhou, X., He, X., and Ren, Y. (2014). Function of microglia and macrophages in secondary damage after spinal cord injury. Neural Regen. Res. 9, 1787-1795. doi: $10.4103 / 1673-5374.143423$

Conflict of Interest Statement: The authors declare that the research was conducted in the absence of any commercial or financial relationships that could be construed as a potential conflict of interest.

Copyright (C) 2017 Umezawa, Naito, Tanaka, Yoshioka, Suzuki, Sudo, Hagihara, Hatano, Tatsumi and Kasuya. This is an open-access article distributed under the terms of the Creative Commons Attribution License (CC BY). The use, distribution or reproduction in other forums is permitted, provided the original author(s) or licensor are credited and that the original publication in this journal is cited, in accordance with accepted academic practice. No use, distribution or reproduction is permitted which does not comply with these terms. 